\title{
Two-frequency operation of a Paul trap to optimise confinement of two species of ions
}

\author{
C. J. Foot ${ }^{\mathrm{a}, *}$, D. Trypogeorgos ${ }^{\mathrm{b}}$, E. Bentine ${ }^{\mathrm{a}}$, A. Gardner ${ }^{\mathrm{c}}$, M. Keller $^{\mathrm{c}}$ \\ ${ }^{a}$ Clarendon Laboratory, Department of Physics, University of Oxford, Parks Road, Oxford, OX1 3PU, UK \\ ${ }^{b}$ Joint Quantum Institute, University of Maryland and National Institute of Standards and Technology, College Park, Maryland, 20742, USA \\ ${ }^{c}$ Department of Physics and Astronomy, University of Sussex, Falmer, BN1 9QH, U.K.
}

\begin{abstract}
We describe the operation of an electrodynamic ion trap in which the electric quadrupole field oscillates at two frequencies. This mode of operation allows simultaneous tight confinement of ions with extremely different mass, e.g., singly ionised atomic ions together with multiply charged nanoparticles. We derive the stability conditions for two-frequency operation from asymptotic properties of the solutions of the Mathieu equation and give a general treatment of the effect of damping on parametric resonances. We show that the two-frequency operation is very effective for a sufficiently large mass ratio between the two species and when the charge of the heavier ions is at least an order of magnitude larger than that of the lighter ones. There is a wide range of conditions for which this system behaves as two 'overlapping' Paul traps which confine ions close to the trap centres allowing both species to interact with each other. This contrasts with existing experiments in single-frequency Paul traps where the more weakly confined species tends to form a sheath around a central core of ions of the other species. We verify these ideas using numerical simulations and by measuring the parametric heating induced in experiments by the additional driving frequency.
\end{abstract}

Keywords:

ion trap, multiple frequencies, parametric heating

The Paul trap $[1,2]$ confines charged particles using an oscillating quadrupole electric field to circumventing Earnshaw's theorem [3] for static fields. This highly versatile method of electrodynamic confinement has found a multitude of applications spanning a wide range of charge-to-mass ratios $(Q / M)$, which may be broadly categorised by the drive frequency of the trap. Guiding of electrons by microwave fields has recently been achieved [4], but practical difficulties of confining them in a Paul trap include driving frequencies of the order of $1 \mathrm{GHz}$. The majority of current experiments to confine atomic ions $\left({ }^{138} \mathrm{Ba}^{+},{ }^{40} \mathrm{Ca}^{+}\right.$, etc.) use a quadrupole electric field oscillating in the range of a few to tens of $\mathrm{MHz}$. Commercial mass spectrometers often have stages in which ions are guided along linear quadrupole fields, operating at $\mathrm{MHz}$ frequencies for light ions, down to tens of $\mathrm{kHz}$ for larger, heavy molecules with reduced $Q / M$. The even lower charge-to-mass ratios of micronsized particles allow these objects to be confined at atmospheric pressure in electrodynamic balances operating at $50 \mathrm{~Hz}[5,6]$. The achievable $Q / M$ typically decreases as the mass of the ion increases, shifting optimal parameters towards lower drive frequencies.

There are many combinations of two species for which the driving frequencies required for trapping are widely separated and applying them simultaneously leads to two separate, overlapping traps applications which in which two ion species with greatly different $Q / M$ ratio must be confined in the same trapping structure. Employing standard Paul traps usually results in

\footnotetext{
${ }^{*}$ Corresponding author

Email address: c.foot@physics.ox.ac.uk (C. J. Foot)
}

very weak trapping of the heavier species and spatial separation of the to ion species in the trapping potential. As a result, the interaction between the two species is weak resulting in reduced detection efficiency of the heavier species as well as its reduced sympathetic cooling by the lighter one.

Pioneering work on the simultaneous trapping of atomic ${ }^{138} \mathrm{Ba}^{+}$and molecular ions with mass $410 \mathrm{Da}$ was carried out using a linear Paul trap operating at a single frequency [7]. A major obstacle for these schemes is a spatial separation of the constituents that occurs when dissimilar charge-to-mass ratio ions, with mismatched spring constants, are confined in the same trap. Ions of the more weakly confined species were pushed away from the trap centre by the Coulomb repulsion from the strongly trapped ions, leading to a sheath of molecular ions around a core of the more tightly confined ${ }^{138} \mathrm{Ba}^{+}$. These heavy ions were indirectly detected by their influence on the spatial distribution of the lighter, fluorescing ions [8]. For mixtures of less similar $Q / M$ the increasing spatial separation between species in a single frequency trap renders this scheme unfeasible.

One aspect that has received little attention is the suggestion by Dehmelt that charged particles with different $Q / M$ can simultaneously be confined by using electric fields oscillating at two suitably chosen frequencies; indeed this may seem counterintuitive for a mass-selective trapping mechanism. Dehmelt proposed the use of two frequencies in a one-page bulletin [9] on confinement of antimatter, e.g., antiprotons and positrons, and this idea has recently been explored further in [10]. In this work, we show that a standard Paul trap for atomic ions 
can be adapted straightforwardly to confine charged particles of higher mass (lower charge-to-mass ratio than atomic ions) using two driving frequencies. The two-frequency trap exploits the implicit link between $Q / M$ of the charged particles and the frequency for practical operation of a Paul trap.

Two-frequency operation of a Paul trap offers a significant advantage when confining two species of different charge-tomass ratios [11], where the trapping potentials lead to a greater proximity of the species. This in turn increases the interaction between the otherwise well separated ion species and thus gives more efficient sympathetic cooling. Here, we demonstrate that this approach is straightforward when the charge-to-mass ratios are widely separated. We solve the system of equations that describes the stability of charged particles in quadrupole electric fields oscillating at two frequencies. This can be interpreted in terms of the pseudopotential approximation that gives an intuitive picture in the single-frequency case. Our calculations of the parametric resonances determine the frequency ratio necessary for stable two-frequency operation with two species. The higher order parametric resonances that lead to instability are very sensitive to damping of the motion $[5,12,13]$ and thus can be easily suppressed by cooling (e.g. laser or buffer gas cooling) as we experimentally demonstrate.

This paper is organised as follows. We start by reviewing the theory of the Paul trap in Section 1 and consider the spring constants for two trapped species. In Section 2 we develop the theory of operation of the two-frequency trap. We discuss parametric resonances and explore the range of applicability of our method. In Section 3 we present experimental results to demonstrate the feasibility of two frequency trapping and compare the results of parametric heating measurements with our theoretical model. In Section 4 we present numerical results from molecular dynamics (MD) simulations showing how the changing spring constant of the weakly trapped species is observable through the deformation the ion cloud in the central core. Finally, we conclude with a discussion of potential applications of our proposed method in Section 5.

\section{Theory of the Paul trap}

Newton's equation of motion for a particle of mass $M$ and electric charge $Q$ along the $y$-axis of a Paul trap is $M(\ddot{y}+\Gamma \dot{y})=$ $Q E_{y}(t)$, where $\Gamma$ is a damping constant. The time-dependent force equals the charge $Q$ times the component of the electric field $E_{y}$ along this axis. The quadrupole field is $E_{y}(t)=$ $V(t) y / r_{0}^{2}$, where $r_{0}$ characterises the electrodes spacing, and the applied voltage is $V(t)=V_{0}+V_{o s c} \cos (\Omega t)$. A change of variables $\Omega t=2 \tau$ transforms the equation of motion into the Mathieu equation $[1,14,15,16]$

$$
\frac{d^{2} y}{d \tau^{2}}+[a-2 q \cos (2 \tau)] y(\tau)=0,
$$

with $a=\left(Q / M \Omega^{2}\right) \cdot 4 V_{0} / r_{0}^{2}$ and $q=-\left(Q / M \Omega^{2}\right) \cdot 2 V_{o s c} / r_{0}^{2}$. An approximate method that elucidates the behaviour of the Paul trap for certain parameters shows that the motion can be considered as an oscillation at a slow secular frequency plus a fast, small-amplitude micromotion at the driving frequency: $y=A \cos (\omega t)(1+q / 2 \cos (\Omega t))$. The slow motion is that of a particle in a harmonic pseudopotential with a secular frequency $\omega$ given by

$$
\omega=\frac{\Omega}{2} \sqrt{a+\frac{1}{2} q^{2}} .
$$

For $a=0$ the motion is stable over the range $0<q<0.91$, as shown in the Ince-Strutt plot of stability regions of the Mathieu equation in Fig. 1. The requirement of stability in both $x$ and $y$ axes is independent of the sign of both $a$ and $q$, and hence the sign of the charge $Q$. Anti-trapping by the static electric field can be overcome by the pseudopotential, e.g., for $q=0.4$ stable solutions exist for $-0.09<a<0.55$. For the value $q \simeq 0.4$ the stable region of $a$ is largest and gives robust operation of a Paul trap. We used this value in Section 2, although the results do not depend on the exact value chosen. Practical considera-

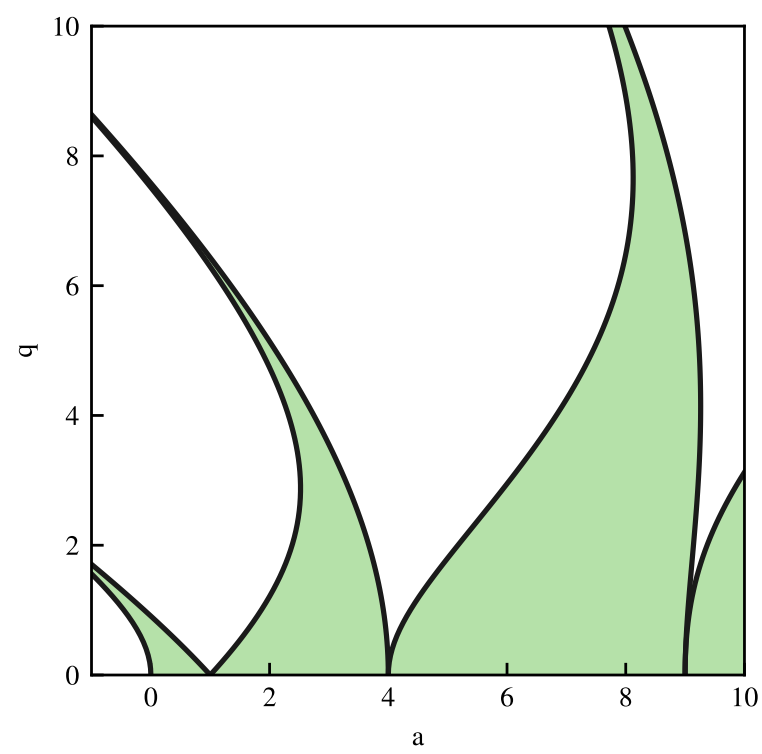

Figure 1: Ince-Strutt stability diagram for the Mathieu equation. The solutions are stable and periodic in the coloured areas and diverging otherwise. Ion traps normally operate in the stability region close to $a=0$ which is widest for $q=0.4$.

tions limit the attainable curvature $V_{\text {max }}^{\prime \prime}=V_{\text {osc }} / r_{0}^{2}$ for a given trap. Realistic values are $V_{\text {osc }}=500 \mathrm{~V}$ and $r_{0}=0.5 \mathrm{~mm}$ giving $V_{\text {max }}^{\prime \prime} \simeq 2 \times 10^{9} \mathrm{~V} / \mathrm{m}^{2}$ which is comparable to the value used in the measurements described in Section 3. This choice of $V_{\max }^{\prime \prime}$ determines the driving frequency that gives $q=0.4$.

\subsection{Confinement of two species in a Paul trap}

We consider two species A, B trapped in a Paul trap as in [11]. Species $\mathrm{A}$ is atomic ${ }^{138} \mathrm{Ba}^{+}$and $\mathrm{B}$ a heavy ion with $M_{B}=1.4 \times 10^{6} \mathrm{Da}$ and $Q_{B}=+33 e$. The spring constant $\kappa=M \omega^{2}$ determines the extent of the ion cloud $y_{\text {rms }}$ at temperature $T$, and hence the overlap of the two species, since by the equipartition theorem $k_{B} T=\kappa y_{\mathrm{rms}}^{2}$. At low $T$ the ions form Coulomb crystals whose spatial extent is determined by 
electrostatic repulsion: equal and opposite trapping forces between two ions $\kappa_{A} y_{A}=-\kappa_{B} y_{B}$ result in similar displacements, $\left|y_{A}\right| \approx\left|y_{B}\right|$, if the spring constants are similar $\kappa_{A} \approx \kappa_{B}$. In the pseudopotential approximation the spring constant is given by

$$
\kappa=M \omega^{2} \approx \frac{1}{8} M q^{2} \Omega^{2}=\frac{Q^{2}}{M \Omega^{2}} \cdot\left(\frac{V_{o s c}}{2 r_{0}^{2}}\right)^{2},
$$

where $\omega \simeq q \Omega / \sqrt{8}$ when $a=0$. The proportionality to $Q^{2} / M$ implies the ratio

$$
\frac{\kappa_{B}(\Omega)}{\kappa_{A}(\Omega)}=\frac{Q_{B}^{2} / M_{B}}{Q_{A}^{2} / M_{A}},
$$

where $\kappa_{A}(\Omega), \kappa_{B}(\Omega)$ are both functions of $\Omega$. For the species considered here $\left(Q_{B}^{2} / M_{B}\right) /\left(Q_{A}^{2} / M_{A}\right)=0.1$, so species B is confined less tightly, thus these ions float to the outside of the cloud in a single-frequency trap. Also $q \propto Q / M$ for fixed $\Omega$, therefore $q_{A}=0.4$ for species A implies $q_{B}=0.001$ so that species $\mathrm{B}$ is only weakly trapped. The spring constant of a Paul trap operating at the optimal frequency depends on the charge only: $\kappa$ is $q / 8$ times the value for a dc quadrupole field with $V_{0}=V_{o s c}$. It follows that this factor of $q / 8$ also applies to the depth of a Paul trap cf., a static quadrupole along one direction (the Dehmelt approximation, Appendix D). In this hypothetical case there would be very strong confinement of species B but species A would be unstable.

\section{Trapping with two frequencies}

We can optimise the confinement of each species individually by using different driving frequencies; a high frequency $\Omega_{H}$ for the light ions and a low frequency $\Omega_{L}$ for the heavier ones. Using the values for the ion species from above as an example, if for $\Omega_{H} / 2 \pi=13 \mathrm{MHz}, q_{A}=\left(Q_{A} / M_{A}\right) \cdot 2 V_{\text {max }}^{\prime \prime} /\left(\Omega_{H}^{2}\right)=0.4$, then the same $q$ parameter can be achieved for the same value of $V_{\max }^{\prime \prime}$ at the lower frequency $\Omega_{L} / 2 \pi=0.8 \mathrm{MHz}$. The ratio of spring constants now becomes

$$
\left[\frac{\kappa_{B}\left(\Omega_{L}\right)}{\kappa_{A}\left(\Omega_{H}\right)}\right]_{\text {overlapping }}=\frac{M_{B}}{M_{A}} \cdot\left(\frac{q_{B} \Omega_{L}}{q_{A} \Omega_{H}}\right)^{2}=\frac{Q_{B}}{Q_{A}} \gg 1 .
$$

In the calculation of these spring constants from the appropriate oscillating quadrupole field we have assumed that the second driving field is not present, i.e., independent operation of the Paul traps, whereas we wish to find out what happens for simultaneous operation with both species in the trap. Although the full improvement between Eq. 4 and Eq. 5 cannot be attained we show that two-frequency operation gives a great improvement over a single frequency as used in previous work [8]. The spring constants for the two species can be made equal (or even be inverted) when two frequencies are applied simultaneously to the electrodes of a quadrupole trap.

\subsection{Parametric resonance}

The stability of mechanical structures against parametrically excited vibrations is closely related to Coulomb crystals of ions subjected to an oscillating potential. The system of two species
A and B subjected to two frequencies $\Omega_{L}$ and $\Omega_{H}$ has a hierarchy of frequencies: $\omega_{B}<\Omega_{L}<\omega_{A}<\Omega_{H}$ as summarised in Table 1.

Table 1: The four relevant frequencies: $\omega_{A}$ is the secular frequency of ions of species A in a Paul trap driven at $\Omega_{H}$. Similarly $\omega_{B}$ for species B with $\Omega_{L}$ only. When both driving frequencies are applied the field at $\Omega_{L}$ can parametrically excite species A but the quadrupole field oscillating at $\Omega_{H}$ only weakly affects species B.

\begin{tabular}{lrr} 
species $\backslash$ rf & $\Omega_{H}$ & $\Omega_{L}$ \\
\hline A & $\omega_{A}$ & parametric excit. \\
B & weak trapping & $\omega_{B}$ \\
\hline
\end{tabular}

The first and third inequality are well satisfied as a consequence of the standard single-frequency operation of a Paul trap; $\omega_{B}=q / \sqrt{8} \cdot \Omega_{L} \simeq 0.14 \Omega_{L}$ and $\omega_{A}=q / \sqrt{8} \cdot \Omega_{H} \simeq$ $0.14 \Omega_{H}$. It is the middle inequality that is important for twofrequency operation; $\Omega_{L}$ must be significantly below $\omega_{A}$ to avoid driving a parametric resonance which leads to heating of species A. The multiple timescales of the system can be untangled in the following way. First, we determine the appropriate amplitude of the voltage oscillating at $\Omega_{H}$ for trapping species A and choose $\Omega_{L}$ such that $\Omega_{L} \ll \omega_{A}$. Next, we calculate the maximum voltage that can be applied at the lower frequency without destabilising species A for a given damping, which occurs when $\Omega_{L}$ drives a parametric resonance of $\omega_{A}$. This voltage will be very small unless $\Omega_{L}$ is sufficiently low. Throughout this paper the higher frequency is taken to be a harmonic $\Omega_{H}=n \Omega_{L}$ of the lower driving frequency, to facilitate the application of the mathematics of periodic systems. The assumption that $n$ is integer makes no difference in the pseudopotential approximation since the physical properties depend on the ratio $\omega_{A} / \Omega_{L}$.

The voltage applied to the quadrupole trap electrodes is $V(t)=V_{0}+V_{1} \cos \left(\Omega_{L} t\right)+V_{n} \cos \left(n \Omega_{L} t\right)$. To consider the stability of species B, we rescale time such that $\Omega_{L} t=2 \tau$ gives

$$
\frac{d^{2} y_{B}}{d \tau^{2}}+\frac{1}{V_{B}}\left(V_{0}+V_{1} \cos (2 \tau)+V_{n} \cos (2 n \tau)\right) y_{B}=0,
$$

where $V_{B}=\left(M_{B} / Q_{B}\right) \cdot \Omega_{L}^{2} r_{0}^{2} / 4$. The dimensionless coefficients are expressed as voltage ratios. This has the form of a Hill equation: $y^{\prime \prime}+H(\tau) y=0$, where $H(\tau)=H(\tau+\pi)$ is a periodic function ${ }^{1}$. The high frequency component oscillating at $\Omega_{H}$ has little effect on the stability of species B, which remains well described by a single-frequency trap operating at the low frequency $\Omega_{L}$.

The same is not true for species A, as parametric resonances lead to heating when $m \Omega_{L}=2 \omega_{A}$, where the integer $m$ is the order of the resonance with $m<n$ since $\omega_{A} \sim 0.14 n \Omega_{L}$. This is clearly seen by considering the equation of motion for species A, rescaled such that $\tilde{\tau}=n \Omega_{L} t / 2=n \tau$

$$
\frac{d^{2} y_{A}}{d \tilde{\tau}^{2}}+\frac{2 \beta}{n} \frac{d y_{A}}{d \tilde{\tau}}+\frac{1}{V_{A}}\left(V_{0}+V_{1} \cos \left(\frac{2 \tilde{\tau}}{n}\right)+V_{n} \cos (2 \tilde{\tau})\right) y_{A}=0,
$$

${ }^{1}$ The Mathieu equation is an example of a Hill equation with only a single periodic coefficient. 
where $V_{A}=\left(M_{A} / Q_{A}\right) \cdot n^{2} \Omega_{L}^{2} r_{0}^{2} / 4$. Note the factor of $n^{2}$ compared to the definition of $V_{B}$ in Eq. 6. The scaled damping parameter $\beta=\Gamma_{A} / \Omega_{L}$ accounts for cooling of species A. For realistic conditions, damping only has a significant effect on the lighter species as can be seen from the appropriate scaling of the equations. The simple mathematical treatment of damping is described in Appendix A. Equations 6 and 7 are ordinary differential equations with periodic coefficients and can be solved in various ways [17]. We have used Floquet theory which considers the mapping from the solution at time $t$ to that at time $t+T$ where $T$ is the period of the system [18]. The influence of any dc field $\propto V_{0}$, the Coulomb repulsion between ions and the damping can be included analytically or by standard numerical methods.

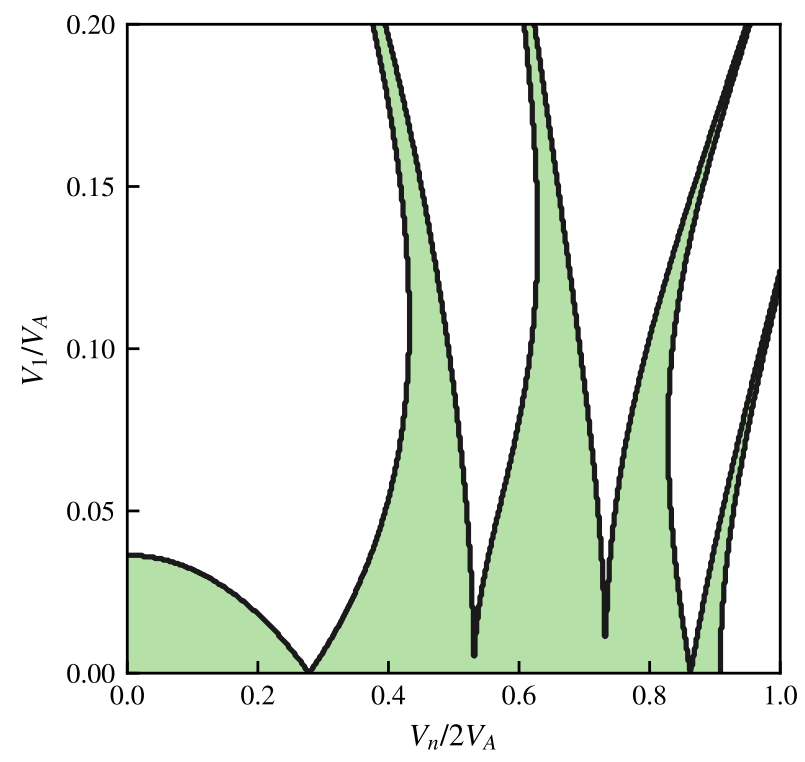

Figure 2: Stability diagram for two-frequency operation given in terms of the applied voltages for $a=0$. Here $\Omega_{H}=5 \Omega_{L}$ leading to four parametric resonances. The tongues of instability don't extend all the way to the horizontal axis due to the finite precision of the numerics. As such, the numerical precision imposes an effective cutoff to the minimum value of the damping parameter $\beta$ that can be included in the numerics.

Figure 2 shows the stability regions for an ion of species $\mathrm{A}$ as a function of $V_{n} / 2 V_{A}$ and $V_{1} / V_{A}$ obtained by numerical solution of Eq. 7 [11]. Parametric excitation by $V_{1} \cos \left(\Omega_{L} t\right)$ causes $n-1$ tongues of instability emanating from the horizontal axis $V_{1}=0$ which get wider as $V_{1}$ increases. The tongues of instability, aka Arnold tongues [19], arise for many periodic systems including the Mathieu equation and their properties have been studied extensively for the more general case of Hill equations [20, 21, 22]. For $n \gtrsim 10$ the stability regions show universal behaviour: a quadratic increase up to a maximum for $V_{n} / 2 V_{A} \simeq 0.7$ followed by an approximately linear decrease to the point $\left(V_{n} / 2 V_{A}, V_{1} / V_{A}\right)=(0.91,0)$. The critical curve that traverses equal distances in the stable and unstable regions $[23,20]$ is a quadratic curve of the form:

$$
\frac{V_{1}}{V_{A}}=\varrho\left(\frac{V_{n}}{2 V_{A}}\right)^{2},
$$

where $\varrho=0.58$ gives the best fit to the numerical calculations of higher order stability diagrams over the range $0<V_{n} / 2 V_{A}<$ 0.71 . This can be explained in terms of the pseudopotential approximation by rewriting Eq. 7 so that it resembles the Mathieu equation assuming $V_{0}=\beta=0$

$$
\frac{d^{2} y_{A}}{d \tilde{\tau}^{2}}+\left[\alpha(\tilde{\tau})-2 q_{A}^{\{\tilde{\tau}\}} \cos 2 \tilde{\tau}\right] y_{A}=0
$$

where $-q_{A}^{\{\tilde{\tau}\}}=V_{n} / 2 V_{A}$ and $\alpha=V_{1} / V_{A}$ resembles an $a$ parameter arising from a dc field but varies in time. However if we assume that the variation of $\alpha(\tilde{\tau})$ is adiabatic then we can use the condition for stability $|\alpha|<q^{2} / 2$. This corresponds to Eq. 8 with $\varrho=0.5$, which is in reasonable agreement with the numerically determined value of 0.58 given above. A more rigorous treatment based on properties of the Mathieu equation for large $a$ and $q$ is given in Appendix A.

The tongues of instability become narrower below the critical line and higher-order resonances are too fine for the numeric calculations to capture - the finite resolution of the numerics acts as an effective damping cutoff. The endpoints of the tongues, for a given damping, can be found from the asymptotic properties of the Mathieu equation and they lie on the curve (see Appendix C)

$$
\frac{V_{1}}{V_{A}}=\rho_{0}\left(\frac{V_{n}}{2 V_{A}}\right)^{2}\left(\frac{\pi \Gamma_{A}}{\Omega_{L}}\right)^{1 / m},
$$

with $\rho_{0}=0.54$. The integer $m$ is the order of the parametric excitation equal to the number of resonant tongues up to the given value of $V_{n} / 2 V_{A}$. The dependence on $\Gamma^{1 / m}$ is expected for the threshold value for parametric excitation of a resonance [24]. The order number $m$ at a resonance can be estimated as

$$
m=\frac{2 \omega_{A}}{\Omega_{L}} \simeq n \frac{q}{\sqrt{2}}=\frac{n}{\sqrt{2}}\left(\frac{V_{n}}{2 V_{A}}\right),
$$

hence $m \simeq 0.28 n$ for $q=0.4$. It has been shown that Eq. 10 gives realistic values for low-order resonances [25, 26]. The width of the instability tongues decreases rapidly with increasing order of the parametric resonance. For the Mathieu equation this width is proportional to $q^{m}$ for the resonance emanating from $(q, a)=\left(0, m^{2}\right)$. Asymptotic approximations work extremely well for narrow resonances as described in Appendix $C$, whereas numerical calculations would require a very fine grid for such narrow features and hence long computation times. Equations 8 and 10 are consistent as $m \rightarrow \infty$, since $\left(\pi \Gamma_{A} / \Omega_{L}\right)^{1 / m} \simeq 1$ for high values of $m$, and $\varrho \simeq \rho_{0}$.

In summary, we chose the voltage $V_{n}$ and frequency $\Omega_{H}=$ $n \Omega_{L}$ to give good confinement of species $\mathrm{A}$ and avoid parametric excitation. Thus Eq. 10 can be considered as a constraint on the three parameters $V_{1}, n$ and $\Gamma_{A}$ noting the implicit dependence of $m$ on $n$ in Eq. 11. Once the values of $V_{1}$ and $n$ that are compatible with the stability of species $\mathrm{A}$ for a given $\Gamma_{A}$ are known, the calculation of the parameters for species B is straightforward. The higher frequency term in Eq. 6, only has a small effect in situations relevant to two-frequency operation; if there was strong confinement with a single frequency then we would not need to consider using two frequencies. 


\subsection{Range of applicability}

In the example described in the previous section we started with a set of parameters suitable for trapping species A namely values of $\left(V_{n}, \Omega_{H}\right)$ that give $q_{A} \simeq 0.4$ in the appropriate Mathieu equation and then determined the voltage $V_{1}$ that can be used to confine species B for given values of $n=\Omega_{H} / \Omega_{L}$ and $\Gamma_{A}$. We chose the 'design criterion' that $\kappa_{B} \geq \kappa_{A}$ which is somewhat arbitrary since it applies to a hypothetical case of one ion of each species in an isotropic trap which is not the case for a linear Paul trap (see Appendix D). Nevertheless balancing the spring constants provides physical insight and guidance in the choice of realistic starting values for MD simulations. Using the criterion $\kappa_{B} \geq \kappa_{A}$, Eq. 5 implies that

$$
\xi=\frac{\kappa_{B}}{\kappa_{A}}=\frac{Q_{B}^{2} / M_{B}}{Q_{A}^{2} / M_{A}} \geq\left(\frac{V_{n}}{n V_{1}}\right)^{2}
$$

We get the ratio of the voltages $V_{1} / V_{n}$ from Eq. 10 as

$$
\frac{V_{1}}{V_{n}}=\frac{\rho_{0}}{2}\left(\frac{V_{n}}{2 V_{A}}\right)\left(\frac{\pi \Gamma_{A}}{\Omega_{L}}\right)^{1 / m}=\frac{1}{2} \rho_{0} q_{A}(\pi \beta)^{1 / m},
$$

where $m$ is the order number and $\beta=\Gamma_{A} / \Omega_{L}$. The function

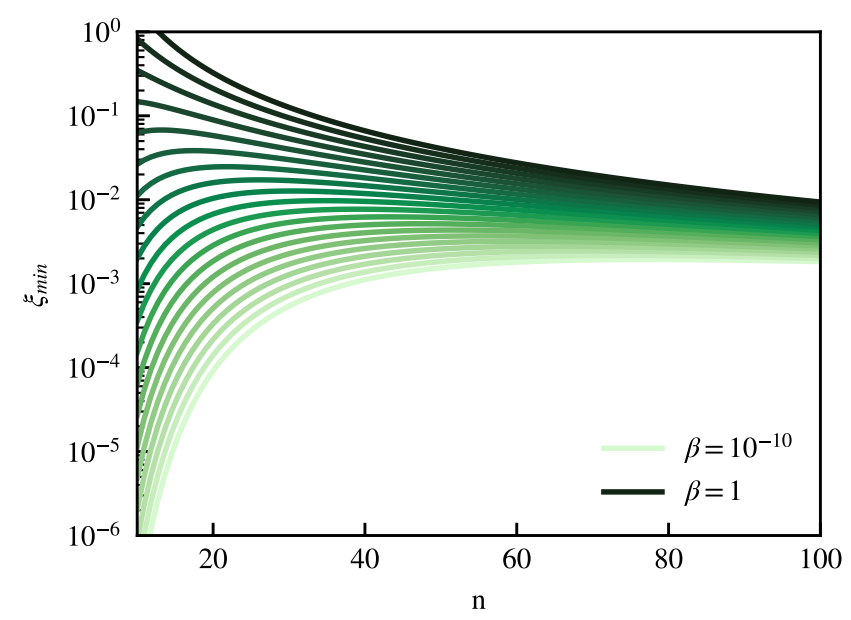

Figure 3: The minimum value $\xi_{\min }$ of the ratio of $Q^{2} / M$ as defined in Eq. 14, is shown against $n$ for various values of $\beta$. The intersections of these curves with the horizontal axis correspond to the minimum value $n_{\min }$ for each value of $\beta$, where $\xi_{\min }=1$. The two-frequency scheme is useful in the regions bounded by $1 \geq \xi \geq \xi_{\min }$. Single-frequency trapping works well for $\xi \geq 1$.

$(\pi \beta)^{1 / m} \rightarrow 1$ as $m$ increases, and varies slowly for large $m$; values for which $h(\beta, m)=1 / 2$ are given in Table 2 . For larger values of $m$ the function lies in the range $0.5 \leq h \leq 1$. It follows from Eq. 13 that $V_{1} / V_{n} \rightarrow \rho_{0} q_{A} / 2 \simeq 1 / 9.3$ as $m \rightarrow \infty$, since $V_{n} /\left(2 V_{A}\right) \equiv q_{A}=0.4$. Hence Eq. 12 becomes

$$
\frac{Q_{B}^{2} / M_{B}}{Q_{A}^{2} / M_{A}} \geq \xi_{\min }=\left(\frac{9.3}{n}\right)^{2}(\pi \beta)^{-7.1 / n},
$$

assuming the approximate relation $m=n / 3.57$ from Eq. 11 with $q_{A}=0.4$. Figure 3 shows $\xi_{\min }$ as a function of $n$ for various values of $\beta$. For $\xi \leq \xi_{\min }$ two-frequency operation can still
Table 2: The order number $m$ and damping parameter $\beta$ for which $h(\beta, m)=$ $1 / 2$ are given by $m=-(\log \beta+\log \pi) / \log 2$. Lower order resonances require increasingly larger damping to be supressed effectively.

\begin{tabular}{rrrrrr}
\hline$\beta$ & $10^{-2}$ & $10^{-4}$ & $10^{-6}$ & $10^{-8}$ & $10^{-10}$ \\
$\mathrm{~m}$ & 5.0 & 11.6 & 18.3 & 24.9 & 31.6 \\
\hline
\end{tabular}

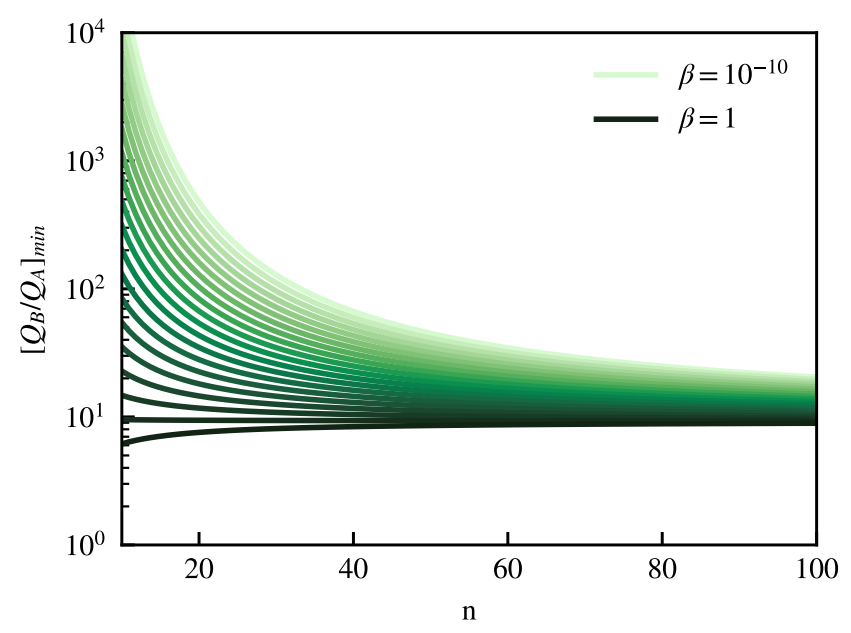

Figure 4: The minimum ratio of the charges $Q_{B} / Q_{A}$ against $n$ for various values of $\beta$, where $\left[Q_{B} / Q_{A}\right]_{\mathrm{min}} \simeq 9.3(\pi \beta)^{-1 / m}$ from Eq. 19 with order number $m \simeq$ $n / 3.6$ estimated from Eq. 11 . The ratio converges to a constant as the order of the resonance approaches infinity.

improve the confinement of the heavier species as in the case of antiprotons and positrons discussed in [9]. The condition $q_{B} \leq 0.4$ must also hold where

$$
q_{B}=\frac{V_{1}}{2 V_{B}}=\frac{V_{A}}{V_{B}} \cdot \frac{V_{n}}{2 V_{A}} \cdot \frac{V_{1}}{V_{n}} .
$$

The maximum value of $V_{1}$ in Eq. 10 determines the confinement of species $\mathrm{B}$ through the parameter

$$
q_{B}=\frac{V_{1}}{2 V_{B}}=\frac{V_{A}}{V_{B}} \cdot \frac{\rho_{0}}{2}\left(\frac{V_{n}}{2 V_{A}}\right)^{2}\left(\frac{\pi \Gamma_{A}}{\Omega_{L}}\right)^{1 / m},
$$

where

$$
\frac{V_{A}}{V_{B}}=\frac{M_{A} / Q_{A}}{M_{B} / Q_{B}} \cdot n^{2}
$$

Here $V_{n} / 2 V_{A}=q_{A}^{\{\tilde{\tau}\}}$ and we assume $q_{B}^{\{\tau\}}=q_{A}^{\{\tilde{\tau}\}}=0.4$ for optimum confinement, thus these factors of $q$ cancel out in Eq. 15 and using Eq. 17 yields

$$
\frac{Q_{B} / M_{B}}{Q_{A} / M_{A}}=\frac{V_{n}}{n^{2} V_{1}} .
$$

Combining this result with Eq. 14 and Eq. 12 gives the minimum ratio of the charges

$$
\frac{Q_{B}}{Q_{A}} \geq 9.3(\pi \beta)^{-3.6 / n},
$$

and masses $M_{B} / M_{A}=n^{2}$. The ratio of the charges is shown in Fig. 4 for various values of $\beta$. 


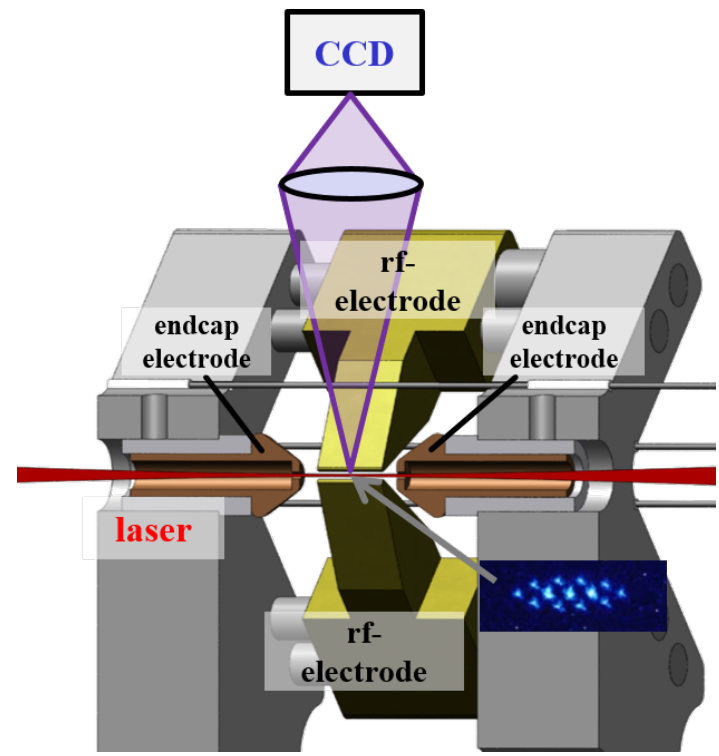

Figure 5: The linear ion trap apparatus used for measuring the parametric resonances. The figure shows a cut through the trapping structure to expose the details of the rf and endcap electrodes. The trapped ions are imaged using a microscope lens and a EMCCD camera at an imaging direction perpendicular to the axis of the trap. A typical image of an ion crystal is shown in the inset.

The limit $\left[Q_{B} / Q_{A}\right]_{\min } \rightarrow 9.3$ as $n \rightarrow \infty$ agrees with the pseudopotential approximation $a \leq q^{2} / 2$ which implies that, for species A,

$$
\frac{a}{2 q}=\frac{V_{1}}{V_{n}} \leq \frac{q}{4}=0.1
$$

hence $Q_{B} \geq 10 Q_{A}$. Thus, we have found a lower limit for the mass ratio $M_{B} \geq n_{\min }^{2} M_{A}$, but more importantly there is no upper limit other than the practicalities of electrodynamic trapping.

The two-frequency scheme works better as the two species become more different in their masses and charge-to-mass ratios. Consider a system of two species with masses in the ratio $M_{B} / M_{A}=10^{4}$ and a ratio of frequencies $\Omega_{H} / \Omega_{L}=n=100=$ $\sqrt{M_{B} / M_{A}}$. The parametric resonance is order $m=28$ and hence for $\beta=10^{-6}$ the function in Eq. 13 has the numerical value $(\pi \beta)^{1 / m}=0.64$. Equation 19 gives $Q_{B} / Q_{A} \simeq 15$. If species $\mathrm{A}$ is ${ }^{138} \mathrm{Ba}^{+}$then charged particles of mass $M_{B}=1.4 \times 10^{6} \mathrm{Da}$ and charge $Q_{B}=+15 e$ can be confined with the same spring constant by simultaneously applying two frequencies to the electrodes of a Paul trap. Keeping the mass the same but increasing the charge to $Q_{B}=+33 e$, we find $\kappa_{B} \simeq 5 \kappa_{A}$. Reworking this calculation for a system with an even higher mass ratio $M_{B} / M_{A}=10^{6}$ and $\Omega_{H} / \Omega_{L}=n=1000=\sqrt{M_{B} / M_{A}}$ we find $h(m, \beta)=h\left(280,10^{-6}\right)=0.94$ and $Q_{B} / Q_{A} \simeq 10$. Thus for this case, and even larger mass and frequency ratios parametric resonances, have little influence which is to be expected since resonances of order $m \gg 10$ are normally weak.

\section{Experimental measurements}

We have carried out experimental measurements with ${ }^{40} \mathrm{Ca}^{+}$ ions confined in a linear Paul trap to test the effect of parametric resonances on the stability of the atomic ions. Our Paul trap has a standard configuration of four parallel rod electrodes creating the quadrupole field operating with a single frequency $\Omega_{H}$. Figure 5 shows he details of the apparatus. The rf electrodes are $4 \mathrm{~mm}$ long with an ion-electrode separation of $0.46 \mathrm{~mm}$. The axial confinement is provided by two endcap electrodes symmetrically placed around the trap center by $2.5 \mathrm{~mm}$ along the trap axis. To provide axial optical access, the electrodes have a hole in their center. The trap is driven asymmetrically, by applying the rf voltage to two opposing electrodes while connecting the other two electrodes to rf ground (see Figure 6). Using the trap electrodes as a capacitance of a resonance circuit, the rf drive voltage is provided by supplying a low-voltage rf drive to a tap of a transformer coil. To allow the application of dc voltages to the rf electrodes for compensating static electric fields, the rf drive is supplied through a capacitor, decoupling the rf drive circuit from the dc voltages. The compensation voltage is supplied through a $1 \mathrm{M} \Omega$ resistor which decouples the dc supply from the rf drive without deteriorating the $\mathrm{Q}$-factor $(\mathrm{Q}=50)$ of the rf circuit.

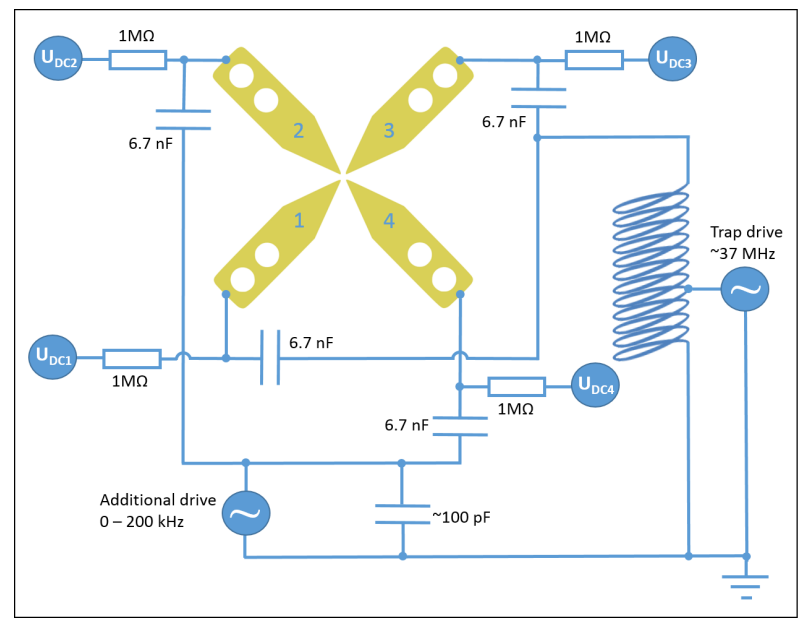

Figure 6: Circuit for two frequency operation of the ion trap. Two of the rf electrodes are driven at a high frequency whereas the second electrode pair is used for the low frequency drive. The dc and rf drives are decoupled using a series of capacitors.

The radial secular oscillation frequency of the ${ }^{40} \mathrm{Ca}^{+}$ions is $\omega_{A} / 2 \pi=1.35 \mathrm{MHz}$ corresponding to $q=0.1$. The axial secular frequency is $0.483 \mathrm{MHz}$ corresponding to $a=3.2 \times 10^{-4}$ for a static voltage of $600 \mathrm{~V}$ on the end-cap electrodes. The amplitude of the applied voltage at $\Omega_{H} / 2 \pi=37.49 \mathrm{MHz}$ deduced from the value of $q$ and an ion-electrode distance of $0.46 \mathrm{~mm}$ is $V_{o s c}=250 \mathrm{~V}$. Two-frequency trapping is achieved by applying a low frequency, low voltage drive to the rf ground electrodes. Using a $100 \mathrm{pF}$ capacitor across the low frequency drive provides a low impedance ground connection for the high frequency trap drive.

Our experiments started with loading a single ${ }^{40} \mathrm{Ca}^{+}$ion into the ion trap. The measurements consisted of imaging the ion onto an EMCCD camera by capturing the fluorescent photons emitted when we excited the resonance transition with laser radiation at a wavelength of $397 \mathrm{~nm}$. Throughout the measure- 

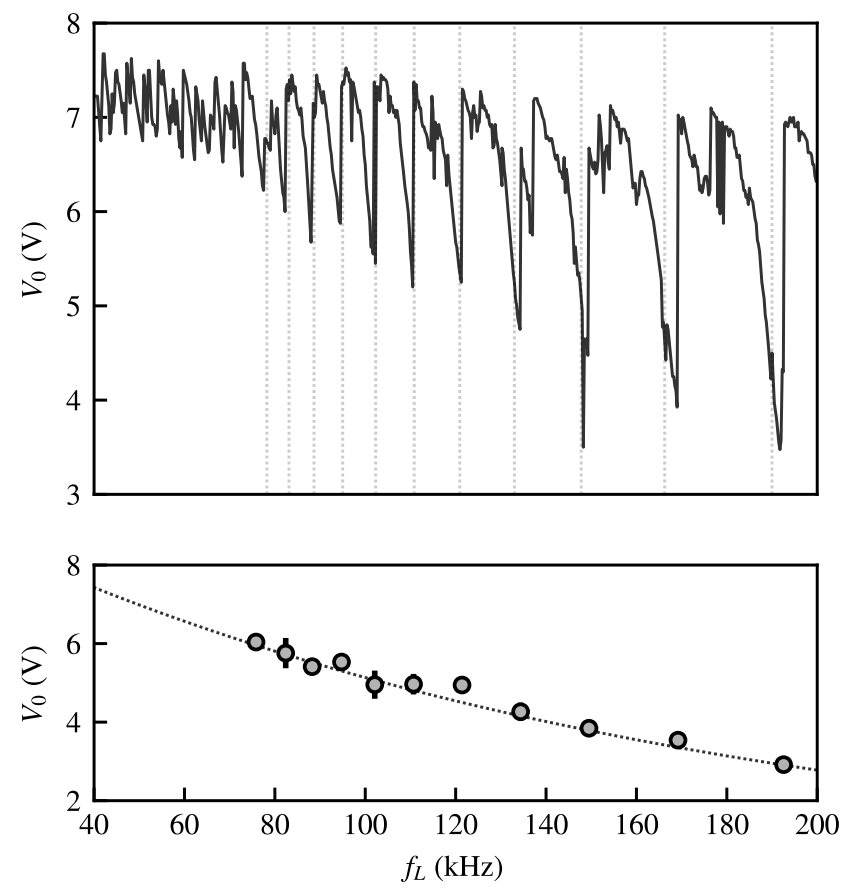

Figure 7: Measured values of the threshold voltage vs the excitation frequency. Twelve distinct resonances can be seen, labeled by their respective order number $m=\{14,16,18,20, \ldots, 34\}$, corresponding to even orders. The higher order resonances, at lower frequencies, fade into the background noise. The solid dots are the line centres of the parametric resonances and the red line is a curve fit of $V_{0} \gamma^{1 / m}$ to the threshold voltage of the resonances. The best-fit parameters are $V_{0}=9.3 \mathrm{~V}$ for $\beta=10^{-6}$ however the the maximum recorded threshold is $7.7 \mathrm{~V}$ as $f \rightarrow 0$.

ments the ion was laser cooled close to the Doppler temperature when there was no parametric excitation. We applied the lower frequency field and increased its amplitude until the Gaussian width of the fluorescent image of the ion increased to five pixels on the camera due to the ion's motion; a single pixel corresponded to a displacement of the ion by $1.32 \mu \mathrm{m}$; the cut-off width of five pixels was chosen to be well above the background fluctuations of the ion's position. This way we were able to take measurements without expelling the ion from the trap each time. For each amplitude, we took two images and averaged their fitted widths along the $x$ and $y$ directions.

The maximum voltage $V_{L}$ corresponding to the cut-off width is shown in Fig. 7 for the range $\Omega_{L} / 2 \pi=40-200 \mathrm{kHz}$. There are twelve distinct resonances in this range, that correspond to even orders $m=\{14,16, \ldots, 34\}$. The threshold for parametric excitation with the applied voltage $V_{L}$ increases as $\Omega_{L}$ decreases. We used Eq. 13 to fit the peaks of the resonances and determine values of $V_{0}$ and $\gamma$. The theoretical limiting voltage $V_{0}$ as $m \rightarrow \infty$ is $V_{0}=0.27 q_{A} V_{n}=7 \mathrm{~V}$ for $q_{A}=0.1$ and $V_{n}=250 \mathrm{~V}$. This is close to the maximum threshold that was measured $7.7 \mathrm{~V}$ but less than the best fit value of $V_{0}=9.3 \mathrm{~V}$. We have not made any corrections for the effect of the finite value of $a$ arising from the dc voltage applied to the end-cap electrodes. These measurements show that a voltage sufficient to confine the heavy ions can be applied in this $\Omega_{L}$ range for
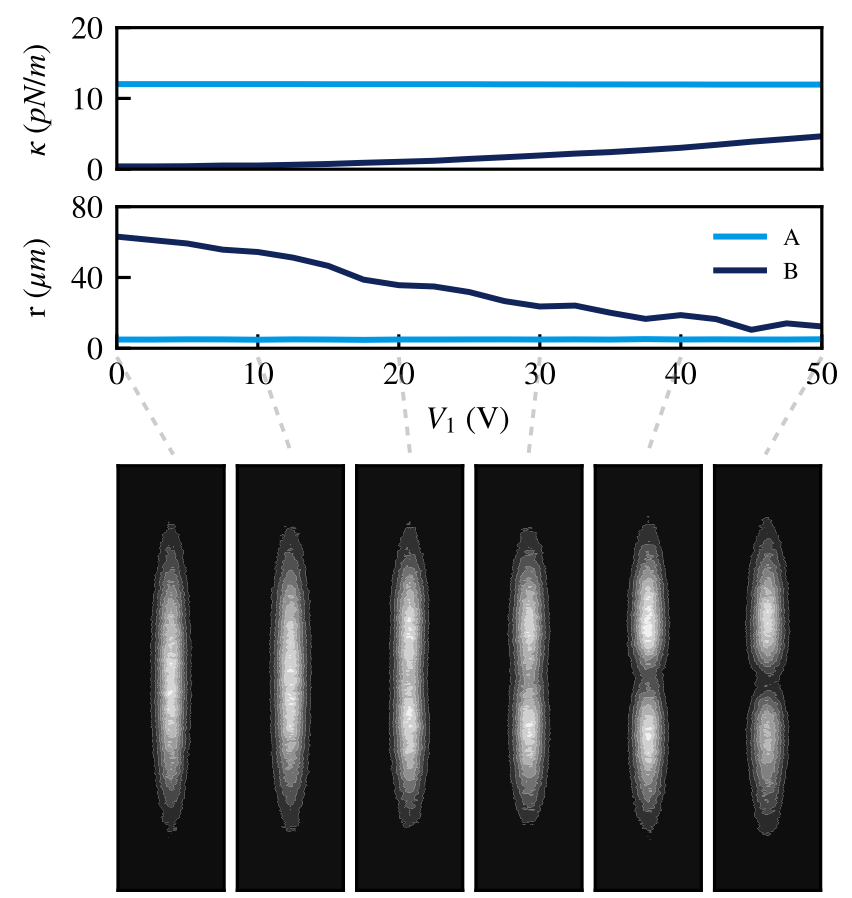

Figure 8: A series of MD simulations shows how the heavy ions are pulled towards the centre of the trap as the voltage of the low-frequency drive increases. The top two panels show the spring constants for the two species and their mean cloud radius. The bottom panel shows the density distribution of the fluorescing light species that develops a hole as the more massive ion displaces the lighter ones.

\section{$V_{L}<7 \mathrm{~V}$.}

Figure 7 al so shows that parametric resonances can be avoided by a suitable choice of $\Omega_{L}$, although the secular oscillation frequencies of species A may be shifted by the presence of species B. For higher values of $m$, the resonances are so weak that their influence becomes comparable with the nonresonant effect of an additional dc voltage. Thus, atomic ions are confined stably in a Paul trap while another quadrupole field oscillating at a lower frequency is applied.

\section{Molecular dynamics simulations}

We have carried out extensive numerical simulations to confirm the stability of multiple ions in the two-frequency trap in all three dimensions. Using the previous single-ion analysis as a guide we simulated the full dynamics of the equations of motion for $\mathrm{N}$ ions. The equation of motion is

$$
M_{j} \ddot{\mathbf{x}}_{j}=\left(E\left(\mathbf{x}_{j} ; t\right)+E_{\perp}\right) Q_{j}+\sum_{\substack{i, j=1 \\ i \neq j}}^{N} \frac{1}{8 \pi \epsilon_{0}} \frac{Q_{i} Q_{j}}{\left(\mathbf{x}_{j}-\mathbf{x}_{i}\right)^{2}}
$$

for ion $j$, where $E\left(\mathbf{x}_{j} ; t\right)$ is the electric field arising from the two-frequency potential $V(t)=V_{0}+V_{1} \cos \Omega_{L} t+V_{n} \cos \Omega_{H} t$, $E_{\perp}$ is an optional transverse electric field and $\epsilon_{0}$ is the permittivity of vacuum. The cutoff range for the included Coulomb potential is much larger than the size of the system so that all 
ions interact with each other. Damping and finite temperature effects are included by coupling the ions to a Langevin bath, analogous to buffer gas cooling, thereby reducing the energy of each particle by a given faction at every time-step [27]. The software tools that we have developed can be used to investigate electrodynamic trapping of multiple species in many different situations even when the timescales for the motion of two species are very different. They provide a light wrapper around LAMMPS [28] ${ }^{2}$, exposing only the relevant subset of its capabilities concerning the simulation of ion trap dynamics [29]. For these MD simulations we modelled the preparation of mixed-species Coulomb crystals starting from thermal clouds well above the transition temperature since it is not sufficient to consider just the final state of the system.

The specific example used in Section 2 shows that applying two frequencies is useful for the particular cases of ${ }^{138} \mathrm{Ba}^{+}$cotrapped with much heavier ions of species B. Applying a field at the lower frequency pulls the heavier ions into the cloud of lighter ions. This overlap is achievable only for certain values of $\left(Q_{B}, M_{B}\right)$ since the strength of the confinement arising from the field produced by the applied voltage $V_{1} \cos \left(\Omega_{L} t\right)$ is limited by the condition that there is no parametric heating of species A as given by Eq. 10. The crystallisation behaviour of ions in this configuration depends on the spring constants for the two species for motion along the axis $\kappa_{\{A, B\} z}=Q_{\{A, B\}} V_{0}^{\{z\}} / z_{0}^{2}$. The maximum value of $V_{1}$ in Eq. 10 determines the confinement of species B through the parameter $q_{B}$. Using Eq. 5 we find a range of voltages $V_{1}$ where the two-frequency trap is stable but the ratio of the spring constants is dramatically different as shown in Fig. 8. For a modest voltage of $50 \mathrm{~V}$ the mean position of the heavy ion is very close to that of the lighter ones and at least an order of magnitude smaller than when $V_{1}=0$. The presence of the heavy ion inside a cloud of lighter ones can be directly detected by the hole the appears in the fluorescence images.

\section{Conclusions and outlook}

The idea of applying a voltage oscillating at two frequencies to the electrodes of a Paul trap has been known by cognoscenti for many years but its application to the simultaneous trapping of two species of charged particles has not yet been realised. Our approach provides a guide to finding suitable operating conditions within the multidimensional parameter space. We have verified the predicted stability of the atomic ions experimentally and carried out numeric simulations of the twofrequency, two-species system. We have shown that the application of a second (lower) frequency to the electrodes of a Paul trap, or ion guide, can easily be implemented on an existing apparatus and the method has very general applicability. This unexploited mode of electrodynamic trapping with quadrupole fields oscillating at two, or more, frequencies greatly extends the capabilities of the Paul trap.

${ }^{2}$ LAMMPS is a classical molecular dynamics code developed by Sandia Labs and distributed as open-source software.
Electrodynamic confinement has many uses ranging from mass spectrometry of small molecules to the control of particles of dimensions hundreds of micrometres. We consider two broad categories of prospective applications: detection of heavy ions via their effect on fluorescing atomic ions, and reactions of cold molecular ions in cold (bio-)chemistry.

The use of a cloud of fluorescing atomic ions to detect a heavy ion is illustrated in Fig. 8 where the hole created by the 'dark ion' is clearly observable. The detection of ions via their effect on atomic ions being excited with laser light is widely used for dark ions such as molecules, or atomic ions with transitions at inconvenient wavelengths. Our results show how to extend this detection technique to massive ions with lower charge-to-mass ratios than atomic ions. This can be used in mass spectrometry for the non-destructive detection of individual molecular ions at a low count rate. Moreover, an intrinsic part of mass spectrometry is fragmentation, i.e., observing the breaking apart of a large biological complex, and the two-frequency scheme enables such investigation of single biomolecular complexes without ensemble averaging. The efficient non-destructive detection of biomolecular ions could be followed by controlled deposition of mass-selected particles on a surface for further analysis by other techniques. It has also been suggested that a chain of atomic and molecular ions can act as a conveyor belt to carry the dark molecular ions into the focus of an x-ray laser for destructive measurements [30]. That proposal was demonstrated by experiments using $\mathrm{Mg}^{+}$and $\mathrm{MgH}^{+}$which have similar charge-to-mass ratios. Our scheme opens the way to working with much heavier biomolecular ions that are of great current interest for single-shot x-ray diffraction experiments.

Paul traps are used to investigate chemical reactions of ions at low temperatures and two-frequency operation allows much heavier species to be used. This application is closely related to the use of two frequencies for the confinement of antimatter where the creation of overlapping clouds of positrons and antiprotons leads to the formation of anti-hydrogen atoms; work towards this is technically challenging goal is ongoing [10]; sympathetic cooling of positrons by $\mathrm{Be}^{+}$ions has been demonstrated experimentally in a Penning trap [31]. Sympathetic cooling of biomolecular ions by ${ }^{138} \mathrm{Ba}^{+}$in a Paul trap was demonstrated [7] and heavier ions can be used by applying two frequencies. The transfer of energy between ions is less efficient if the oscillation frequencies are dissimilar. However there is coupling between axial modes of species $\mathrm{A}$ and radial modes of species of species B in a linear Paul trap which has a large difference in the frequencies of oscillations in these directions. The extension of the techniques developed for lasercooled atomic ions to species with much higher $M / Z$ presents new ground for future investigation. Flakes of graphite and graphene have also been confined in a Paul trap [32]. Moreover, the confinement of DNA in a Paul trap has been proposed and numerically simulated in which the long chain-like biomolecules lying along the axis of a quadrupole in aqueous solution rather than the usual vacuum environment [33]. 


\section{Acknowledgements}

The authors would like to thank...

\section{Appendix A. The mathematical treatment of damping}

Equations of motion with damping proportional to velocity, or any second-order differential equation in which the first and second derivatives have constant coefficients, can be written in the general form $\ddot{y}+\Gamma \dot{y}+H(t) y=0$. The substitution $y=\tilde{y} \exp (-\Gamma t / 2)$ eliminates the term with the first derivative to give $\ddot{\tilde{y}}+\tilde{H}(t) \tilde{y}=0$, where $\tilde{H}(t)=H(t)-\Gamma^{2} / 4$. Secondorder equations with linear damping have an associated equation without damping [REF] which is a Hill equation when $\tilde{H}(t)$ is a periodic function and can be written as the sum of its Fourier components. An illustrative example is the damped simple harmonic oscillator with a parametric driving term

$$
\frac{d^{2} y}{d t^{2}}+\tilde{\omega}_{A}^{2} y=\frac{F^{\prime}}{M_{A}} \cos (\Omega t) y
$$

where $F^{\prime}$ is a constant and the secular oscillation frequency is modified to $\tilde{\omega}_{A}^{2}=\omega_{A}^{2}-\Gamma^{2} / 4$. This is the usual shift of the resonance frequency that occurs in damped simple harmonic motion. Note however, that the integrating factor $\exp (-\Gamma t / 2)$ eliminates $\dot{y}$ for any value of the constant $\Gamma$ and is used in numerical calculations of the stability regions of the Mathieu equation with strong damping $[5,12]$.

\section{Appendix B. Interpretation in terms of the Mathieu equa- tion}

To relate the behaviour of ions in a quadrupole electric field oscillating at two frequencies (including a dc term) to familiar properties of a single-frequency Paul trap we consider Eq. 7 with $V_{1}=0$ which reduces to a Mathieu equation with parameter

$$
q_{A}^{\{\tilde{\tau}\}}=\frac{V_{n}}{2 V_{A}}=\frac{Q_{A}}{M_{A}} \cdot \frac{4 V_{n}^{\prime \prime}}{(n \Omega)^{2}},
$$

where $V_{n}^{\prime \prime}=V_{n} / r_{0}^{2}$ is the curvature of the oscillating electric potential. We drop the minus sign since the steady-state behaviour does not depend on the sign of $q$. We take $q_{A}^{\{\tilde{\tau}\}}=0.4$ as a realistic value for operation of a Paul trap since for higher values the ions are susceptible to heating and loss from nonlinear mixing and parametric excitation even for single frequency operation.

The quadrupole field proportional to $V_{n} \cos n \Omega t$ leads to a pseudopotential with a secular oscillation frequency $\omega_{A}=$ $q_{A}^{\{\tilde{\tau}\}} n \Omega / \sqrt{8}$ so ions of species A undergo simple harmonic motion described by $\ddot{y}+\omega_{A}^{2} y=0$ where $t$ is real time. We introduce a voltage $V_{1} \cos \Omega t$ that produces a driving term proportional to $F^{\prime}=Q_{A} V_{1} / r_{0}^{2}$ in Eq. A.1. Using the rescaling $\Omega t=2 \tau$ as in Eq. 6 gives

$$
\frac{d^{2} y}{d \tau^{2}}+2 \beta \frac{d y}{d \tau}+\left(a_{\mathrm{eff}}-2 q_{A}^{\{\tau\}} \cos 2 \tau\right) y=0,
$$

where $\beta=\Gamma_{A} / \Omega$ and

$$
a_{\mathrm{eff}}=\left(\frac{2 \omega_{A}}{\Omega}\right)^{2}=\frac{1}{2}\left(\frac{n V_{n}}{2 V_{A}}\right)^{2}=\frac{1}{2}\left(n q_{A}^{\{\tilde{\tau}\}}\right)^{2}
$$

is the effective value of a static potential equivalent to the pseudopotential (with $V_{0}=0$ ), and

$$
-q_{A}^{\{\tau\}}=\frac{V_{1} n^{2}}{2 V_{A}}
$$

is completely independent from $q_{A}^{\{\tilde{\tau}\}}$. For $q_{A}^{\{\tilde{\tau}\}}=0.4$ and $n=100$ we find that $a_{\mathrm{eff}}=800$. This is far greater than is usually considered in the theory of Paul traps but the asymptotic properties of the Mathieu equation are known in literature [34, 23]. For $a, q \gg 1$ the critical line between predominantly stable and unstable regions is $a=2 q$. This passes through the point $(q, a)=(400,800)$ in this example. From $a_{\mathrm{eff}}=2\left|q_{A}^{\{\tau\}}\right|$, and Equations B.3, B.4, we recover Eq. 8 with the same value $\varrho=0.5$ as the simplified argument given previously. Damping modifies $a_{\text {eff }}$ however this is not a significant effect for $\Gamma /\left(2 \omega_{A}\right)<0.1$ and is neglected in this paper.

\section{Appendix C. Theory of parametric excitation with linear damping}

The parametric excitation of ions is a standard Paul trap was investigated experimentally and theoretically in $[25,26]$. We extend their results to high order resonances using asymptotic properties of the instability tongues of the Mathieu equation. The threshold voltage above which an applied field at $\Omega$ excites a resonance of order $m$ is

$$
\left[\frac{V_{1}}{r_{0}^{2}}\right]_{\mathrm{th}}=\frac{2 M \omega_{A}^{2}}{Q} C_{m}\left(\frac{\Gamma}{2 \omega}\right)^{1 / m}
$$

with $M \equiv M_{A}, Q \equiv Q_{A}$ and $\Gamma \equiv \Gamma_{A}$ here. This corresponds to Eq. 10 with $\rho_{0}=(2 / \mathrm{e})^{2} \simeq 0.54$ for

$$
C_{m}=\left(\frac{2}{e}\right)^{2}(\pi m)^{1 / m}
$$

This formula for $C_{m}$ is derived below by relating the damping to the width of tongues of instability. Razvi et al. estimated the coefficient to be $C_{m} \simeq 2$ from numerical calculations of the first few resonances. Zhao et al. find the values $\left\{C_{1}, C_{2}, \ldots C_{6}\right\}=$ $\{2,1.414, \ldots 0.887\}$ whereas Eq. C. 2 gives $\{1.7,1.36, \ldots 0.887\}$. Thus our general formula is a good approximation for the width of resonances except for $m=1$. Importantly it gives an analytic expression for the high orders $m \gg 10$ relevant to our twofrequency scheme.

The following derivation uses notation similar to Zhao et al. to highlight similarities and differences. The damping constant $\beta=\Gamma / \Omega$ can be eliminated from Eq. B.2 by the substitution $y=\tilde{y} \exp (-\beta \tau)$ to give the Mathieu equation

$$
\frac{d^{2} \tilde{y}}{d \tau^{2}}+\left(\tilde{a}_{\mathrm{eff}}-2 q_{A}^{\{\tau\}} \cos 2 \tau\right) \tilde{y}=0
$$


where $\tilde{a}_{\mathrm{eff}}=\left(a_{\mathrm{eff}}-\beta^{2}\right)$ hence

$$
\tilde{a}_{\mathrm{eff}}=\left(\frac{2 \omega_{A}}{\Omega}\right)^{2}\left(1-\gamma^{2}\right)=\left(\frac{2 \tilde{\omega}_{A}}{\Omega}\right)^{2},
$$

with $\gamma=\Gamma /\left(2 \omega_{A}\right) \ll 1$ and $\tilde{\omega}_{A}=\omega_{A} \sqrt{1-\gamma^{2}}$. We neglect the slight frequency shift $\left(\omega_{A}-\tilde{\omega}_{A}\right)$ because $\gamma^{2} \ll 1$. Using Eq. 11 we find

$$
q_{A}^{\{\tau\}}=\frac{2 Q V_{1}}{M r_{0}^{2} \Omega^{2}}=\epsilon\left(\frac{2 \omega_{A}}{\Omega}\right)^{2}=\epsilon m^{2}
$$

is unaffected by damping, and we have defined

$$
\epsilon=\frac{Q V_{1}}{2 M r_{0}^{2} \omega_{A}^{2}}
$$

Floquet's theorem states that equations with periodic coefficients have solutions of the form $\tilde{y}=e^{\mu \tau} u(\tau)$ where $u$ is a periodic function; in this case $u(\tau)=u(\tau+\pi)$ to match the period of $\cos 2 \tau$. The solution of Eq. C. 3 is a linear combination of two such independent functions. Hence the solutions in real time $t$ have the form

$$
y=C_{1} e^{(\mu \Omega-\Gamma) t / 2} u_{1}(t)+C_{2} e^{-(\mu \Omega+\Gamma) t / 2} u_{2}(t) .
$$

Instability arises if $\mu>\Gamma / \Omega=\gamma m$. The stability of the solution can be determined from the relationship between the characteristic exponent $\mu$ and the width of the instability tongues [REF]. Considering the shape of the tip of the tongues gives

$$
\gamma=\frac{\mu}{m}=\frac{a_{m}^{+}-a_{m}^{-}}{4 m^{2}}
$$

where $a_{m}^{+}(q)$ and $a_{m}^{-}(q)$ are the values at the upper and lower boundary of the $m$ th tongue. The width of the undamped resonance is $\Delta a_{m}=a_{m}^{+}-a_{m}^{-}=A_{m} q^{m}$. From Eq. C.5 we find the excitation threshold as

$$
[\epsilon]_{\mathrm{th}}=\left(\frac{4 m^{2}}{A_{m}}\right)^{1 / m} \frac{\gamma^{1 / m}}{m^{2}}=C_{m} \gamma^{1 / m} .
$$

This is equivalent to Eq. C.1, with

$$
C_{m}=\frac{1}{m^{2}}\left(\frac{4 m^{2}}{A_{m}}\right)^{1 / m} .
$$

Using the asymptotic formula for $A_{m}$ given in [35]

$$
\frac{A_{m}}{4 m^{2}}=\frac{2}{2^{2 m}[m !]^{2}},
$$

and Sterling's formula for $m$ !

$$
A_{m}=\frac{4}{\pi}\left(\frac{\mathrm{e}}{2 m}\right)^{2 m}
$$

leads to Eq. C.2.

We can now use this to examine the validity of the approximations. Resonances occur for $a_{m}=m^{2}$ hence their spacing is approximately $a_{m+1}-a_{m} \simeq 2 m$ for large $m$. Thus the condition that width of the tongues is small compared to their spacing is
$A_{m} q^{m} \ll 2 m$ which implies $q \ll(\pi m / 2)^{1 / m}(2 m / \mathrm{e})^{2}$. This is satisfied if $2 q \ll a=m^{2}$ which corresponds to the expectations that the approximation is valid well away from the critical line where the tongues of instability are narrow. The formula for the width $\Delta a_{m}=a_{m}^{+}-a_{m}^{-}=A_{m} q^{m}$ is not restricted to small values of $q$. Equation 8 is a limiting form of Eq. 10 assuming $\rho_{0} \approx \rho$. For higher damping, $\Gamma>0.1$, other approximations may be useful, or in such cases it is straightforward to carry out numerical calculations since the tongues of instability have rounded ends so that computing their boundary does not require an excessively fine computational grid. In contrast the stability regions reaching into the region $2 q>a$ are cusped even for strong damping $[\mathrm{REF}]$ but these are not relevant here.

\section{Appendix D. Two species in a linear Paul trap}

A linear Paul trap has four electrodes aligned parallel to the z-axis arranged on the corners of a square in the xy-plane, with adjacent electrodes having opposite polarity in a quadrupole configuration. The oscillating electric field has no component of the electric field along $z$ and $E_{y}=V_{\max }^{\prime \prime} y$ then $E_{x}=-V_{\max }^{\prime \prime} x$ where $V_{\max }^{\prime \prime}=V_{o s c} / r_{0}^{2}$ is the curvature. It is assumed that the trap is operated at the maximum voltage in a given apparatus to give the strongest confinement but this might not be optimal, e.g., for very large clouds of ions. Thus in the Mathieu equations describing the motion in the $x$ and $y$ directions respectively $a_{x}=-a_{y}$. If $q=0$ then Eq. 1 simplifies to simple harmonic motion $y^{\prime \prime}=-a y$ at angular frequency $\sqrt{a}$ for $a>0$, and for $a<0$ the motion is unstable. Thus the central axis of the four electrodes is a line of saddle points of the electrostatic potential energy of charged particles commonly referred to as the rf-null line. There can be stable motion when an oscillating voltage is applied to these electrodes. This can be shown using the pseudopotential approximation, valid for small $a$ and $q$ in Mathieu equation, by substituting a trial solution of the form $y=C \cos (\omega t)(1+D \cos (\Omega t))$, where $t$ is real time. Equating terms with the same time dependence leads to Eq. 2. The pseudopotential has an effective depth of $q V_{\text {osc }} / 8$; this is called the Dehmelt approximation [36]. The discussion of radial confinement in the main text can be summarized by expressions for the potential energy of ions along the $y$-axis:

$$
\begin{aligned}
& U_{A}=\left(\kappa_{A}(0)+\kappa_{A}\left(\Omega_{H}\right)\right) y^{2} / 2, \\
& U_{B}=\left(\kappa_{B}(0)+\kappa_{B}\left(\Omega_{L}\right)+\kappa_{B}\left(\Omega_{H}\right)\right) y^{2} / 2, \\
& U_{B} \simeq\left(\kappa_{B}(0)+\kappa_{B}\left(\Omega_{L}\right)\right) y^{2} / 2 .
\end{aligned}
$$

The last line follows because $\kappa_{B}\left(\Omega_{L}\right) \gg \kappa_{B}\left(\Omega_{H}\right)$ in twofrequency operation.

In the main text we considered that the dc terms are negligible: $\kappa_{B}(0) \simeq 0 \simeq \kappa_{A}(0)$. In a linear Paul trap, however, there is an static radial field as a consequence of the axial confinement. An axially symmetric voltage satisfying $\nabla \mathbf{E}=0$ has the form $V=V_{0}^{\{z\}}\left[z^{2}-\frac{1}{2}\left(x^{2}+y^{2}\right)\right] /\left(2 z_{0}^{2}\right)$ hence the axial confinement necessarily acts oppositely in the radial directions, e.g $\kappa_{B}(0) \equiv \kappa_{B, y}(0)=-\kappa_{B, z} / 2$. The static spring constant $\kappa_{B, z}=Q_{B} V_{0}^{\{z\}} / z_{0}^{2}$ depends only on the charge, and similarly 
$\kappa_{A, z}=Q_{A} V_{0}^{\{z\}} / z_{0}^{2}$, which is very different to the dependence on $Q^{2} / M$ for an ac field. The anti-trapping arising from the radial component of the static field is of particular concern for species B which is only weakly confined by a single-frequency ac field, as the following calculation illustrates. Consider a Paul trap with an aspect ratio $\lambda=\omega_{A} / \omega_{A, z}$; this ratio of radial to axial oscillation frequencies for species $\mathrm{A}$ gives the ratio of the length to radius of the elongated cigar-shaped cloud of A-ions in thermal equilibrium $\kappa_{A}\left(\Omega_{H}\right)=\lambda^{2} \kappa_{A, z}$. The requirement that $\kappa_{B}\left(\Omega_{H}\right) \geq \kappa_{B, z} / 2$ gives a stability condition for species $\mathrm{B}$ in a single-frequency linear trap

$$
\frac{Q_{B}}{M_{B}} \geq \frac{1}{2 \lambda^{2}} \cdot \frac{Q_{A}}{M_{A}}
$$

where we used Eq. 4. For two species with $\left(Q_{B} / M_{B}\right) /\left(Q_{A} / M_{A}\right)=3.3 \cdot 10^{-3}$ this gives $\lambda \geq 12.3$. Hence this large aspect ratio is required to reach the edge of the stability region, indicating the difficulty of confining two species of widely different $Q / M$ with a standard Paul trap. Also this underestimates the problem since a cloud of atomic ions of species A collectively exert a stronger repelling force on an ion of species B. The collective effect of many ions of species A should also be taken into account in two-frequency operation since it acts to drive species B out of the trap. Another factor for a linear Paul trap is that there is the weaker confinement along the $z$-axis than radially $\kappa_{A, z}<\kappa_{A, y} \sim \kappa_{A, x}$ and therefore the criterion for species B to lie on the axis is $\kappa_{B, y}>\kappa_{A, z}$. The heavier ion(s) can displace species $A$ along the $z$-axis more easily than radially as shown in Fig. 8. Thus, as stated in the main text, the criterion $\kappa_{B} \geq \kappa_{A}$ which considers only the radial direction(s) is a simplification. These approximate calculations provide useful physical insight; the various competing effects can be studied using MD simulations.

\section{References}

[1] W. Paul, Electromagnetic traps for charged and neutral particles, Rev. Mod. Phys. 62 (3) (1990) 531-540. doi:10.1103/RevModPhys.62.531. URL http://link.aps.org/doi/10.1103/RevModPhys.62.531

[2] W. Paul, Electromagnetic Traps for Charged and Neutral Particles (Nobel Lecture), Angewandte Chemie International Edition in English 29 (7) (1990) 739-748. doi:10.1002/anie.199007391.

[3] S. Earnshaw, On the Nature of the Molecular Forces which Regulate the Constitution of the Luminiferous Ether, Transactions of the Cambridge Philosophical Society 7 (1848) 97.

URL http: //adsabs . harvard.edu/abs/1848TCaPS . . 7 . . 97E

[4] J. Hoffrogge, P. Hommelhoff, Planar microwave structures for electron guiding, New J. Phys. 13 (9) (2011) 095012. doi:10.1088/13672630/13/9/095012.

URL http: //stacks . iop.org/1367-2630/13/i=9/a=095012

[5] M. Nasse, C. Foot, Influence of background pressure on the stability region of a Paul trap, European Journal of Physics 22 (2001) 563-573. doi:10.1088/0143-0807/22/6/301. URL http: //iopscience.iop.org/0143-0807/22/6/301

[6] H. Winter, H. W. Ortjohann, Simple demonstration of storing macroscopic particles in a Paul trap, American Journal of Physics 59 (9) (1991) 807-813. doi:10.1119/1.16830.

URL http://aapt.scitation.org/doi/abs/10.1119/1.16830

[7] D. Offenberg, C. B. Zhang, C. Wellers, B. Roth, S. Schiller, Translational cooling and storage of protonated proteins in an ion trap at subkelvin temperatures, Phys. Rev. A 78 (6) (2008) 061401.
doi:10.1103/PhysRevA.78.061401.

URL http://link.aps.org/doi/10.1103/PhysRevA.78.061401

[8] A. Ostendorf, C. B. Zhang, M. A. Wilson, D. Offenberg, B. Roth, S. Schiller, Sympathetic Cooling of Complex Molecular Ions to Millikelvin Temperatures, Phys. Rev. Lett. 97 (24) (2006) 243005. doi:10.1103/PhysRevLett.97.243005.

URL http://link.aps.org/doi/10.1103/PhysRevLett.97.243005

[9] H. Dehmelt, Economic synthesis and precision spectroscopy of antimolecular hydrogen ions in Paul trap, Phys. Scr. 1995 (T59) (1995) 423. doi:10.1088/0031-8949/1995/T59/060.

URL http://iopscience.iop.org/1402-4896/1995/T59/060

[10] N. Leefer, K. Krimmel, W. Bertsche, D. Budker, J. Fajans, R. Folman, H. Haeffner, F. Schmidt-Kaler, Investigation of two-frequency Paul traps for antihydrogen production, arXiv:1603.09444 [hep-ex, physics:physics]ArXiv: 1603.09444. URL http: //arxiv.org/abs/1603.09444

[11] D. Trypogeorgos, C. J. Foot, Cotrapping different species in ion traps using multiple radio frequencies, Phys. Rev. A 94 (2) (2016) 023609. doi:10.1103/PhysRevA.94.023609.

URL http://link. aps .org/doi/10.1103/PhysRevA .94.023609

[12] T. Hasegawa, K. Uehara, Dynamics of a single particle in a Paul trap in the presence of the damping force, Applied Physics B Laser and Optics 61 (1995) 159-163. doi:10.1007/BF01090937.

URL http: //www . springerlink. com/content/u204512502321413/

[13] P. Pedersen, Stability of the solutions to Mathieu-Hill equations with damping, Ing. arch 49 (1) (1980) 15-29. doi:10.1007/BF00536595. URL http://link. springer. com/article/10.1007/BF00536595

[14] D. J. Berkeland, J. D. Miller, J. C. Bergquist, W. M. Itano, D. J. Wineland, Minimization of ion micromotion in a Paul trap, Journal of Applied Physics 83 (10) (1998) 5025-5033. doi:10.1063/1.367318.

URL http://scitation.aip.org/content/aip/journal/jap/83/10/10.10

[15] D. Wineland, C. Monroe, W. Itano, D. Leibfried, B. King, D. Meekhof, Experimental issues in coherent quantum-state manipulation of trapped atomic ions, Journal of Research of the National Institute of Standards and Technology 103 (3) (1998) 259. doi:10.6028/jres.103.019. URL http://nvlpubs.nist.gov/nistpubs/jres/103/3/j33win.pdf

[16] P. K. Ghosh, Ion Traps, Oxford University Press, Oxford : New York, 1996.

[17] D. Jordan, P. Smith, Nonlinear Ordinary Differential Equations: An Introduction for Scientists and Engineers, 4th Edition, OUP Oxford, 2007.

[18] G. Floquet, Sur les equations differentielles lineaires a coefficients periodiques, Annales scientifiques de l'Ecole Normale Superieure 12 (1883) 47-88.

URL https://eudml .org/doc/80895

[19] V. I. Arnol'd, Mathematical Methods of Classical Mechanics, Springer, 1989.

[20] M. I. Weinstein, J. B. Keller, Asymptotic Behavior of Stability Regions for Hill's Equation, SIAM Journal on Applied Mathematics 47 (5) (1987) 941-958, articleType: research-article / Full publication date: Oct., 1987 / Copyright 1987 Society for Industrial and Applied Mathematics. doi:10.2307/2101700.

URL http://www . jstor .org/stable/2101700

3211] jabstr.ssitnakhina, C. Tier, Computing the stability regions of Hill's equation, Applied Mathematics and Computation 162 (2) (2005) 639-660. doi:10.1016/j.amc.2004.01.002.

URL http://www . sciencedirect.com/science/article/pii/S009630030

[22] L. F. Roncaratti, V. Aquilanti, WhittakerHill equation, Ince polynomials, and molecular torsional modes, International Journal of Quantum Chemistry 110 (3) (2010) 716-730. doi:10.1002/qua.22255. URL http://onlinelibrary. wiley.com/doi/10.1002/qua.22255/abstra

[23] H. Broer, M. Levi, C. Simo, Large scale radial stability density of Hill's equation, Nonlinearity 26 (2) (2013) 565. doi:10.1088/0951$7715 / 26 / 2 / 565$.

URL http: //iopscience.iop.org/0951-7715/26/2/565

[24] L. D. Landau, E. M. Lifshitz, Mechanics: Volume 1, 3rd Edition, Butterworth-Heinemann, Amsterdam u.a, 1976.

[25] M. A. N. Razvi, X. Z. Chu, R. Alheit, G. Werth, R. Blmel, Fractional frequency collective parametric resonances of an ion cloud in a Paul trap, Phys. Rev. A 58 (1) (1998) R34-R37. doi:10.1103/PhysRevA.58.R34. URL http: //link. aps . org/doi/10.1103/PhysRevA . 58.R34

[26] X. Zhao, V. L. Ryjkov, H. A. Schuessler, Parametric excitations of 
trapped ions in a linear rf ion trap, Phys. Rev. A 66 (6) (2002) 063414. doi:10.1103/PhysRevA.66.063414.

URL http://link.aps.org/doi/10.1103/PhysRevA.66.063414

[27] C. B. Zhang, D. Offenberg, B. Roth, M. A. Wilson, S. Schiller, Moleculardynamics simulations of cold single-species and multispecies ion ensembles in a linear Paul trap, Phys. Rev. A 76 (1) (2007) 012719. doi:10.1103/PhysRevA.76.012719.

URL http: //link. aps .org/doi/10.1103/PhysRevA.76.012719

[28] S. Plimpton, Fast Parallel Algorithms for Short-Range Molecular Dynamics, Journal of Computational Physics 117 (1) (1995) 1-19. doi:10.1006/jcph.1995.1039.

URL http: //www.sciencedirect.com/science/article/pii/S002199918571039X

[29] E. Bentine, C. Foot, D. Trypogeorgos, (py)lion an: open source wrapper of LAMMPS for the simulation of trapped ions, in preparation.

[30] S. Kahra, G. Leschhorn, M. Kowalewski, A. Schiffrin, E. Bothschafter, W. Fues, R. de Vivie-Riedle, R. Ernstorfer, F. Krausz, R. Kienberger, T. Schaetz, A molecular conveyor belt by controlled delivery of single molecules into ultrashort laser pulses, Nat Phys 8 (3) (2012) 238-242. doi:10.1038/nphys 2214 .

URL http://www . nature.com/nphys/journal/v8/n3/full/nphys2214.html

[31] B. M. Jelenkovic, A. S. Newbury, J. J. Bollinger, W. M. Itano, T. B. Mitchell, Sympathetically cooled and compressed positron plasma, Phys. Rev. A 67 (6) (2003) 063406. doi:10.1103/PhysRevA.67.063406.

URL http://link.aps.org/doi/10.1103/PhysRevA.67.063406

[32] B. E. Kane, Levitated spinning graphene flakes in an electric quadrupole ion trap, Phys. Rev. B 82 (11) (2010) 115441. doi:10.1103/PhysRevB.82.115441.

URL http://link .aps .org/doi/10.1103/PhysRevB.82.115441

[33] X. Zhao, P. S. Krstic, Molecular dynamics simulation study on trapping ions in a nanoscale Paul trap, Nanotechnology 19 (19) (2008) 195702. doi:10.1088/0957-4484/19/19/195702.

URL http://www.ncbi.nlm.nih.gov/pmc/articles/PMC3269946/

[34] H. Broer, M. Levi, Geometrical aspects of stability theory for Hill's equations, Arch. Rational Mech. Anal. 131 (3) (1995) 225-240. doi:10.1007/BF00382887.

URL http: //link. springer . com/article/10.1007/BF00382887

[35] NIST Handbook of Mathematical Functions | Abstract analysis | Cambridge University Press.

URL http://www. cambridge.org/gb/academic/subjects/mathematics/abstract-analysis/nist-handbook-mathematical-functions

[36] R. E. March, J. F. J. Todd, Quadrupole Ion Trap Mass Spectrometry,

Volume 165, Second Edition, Wiley-VCH, 2005.

URL http://onlinelibrary.wiley.com/book/10.1002/0471717983/homepage/Reviews.html 